\title{
Sliding Mode Control for Singularly Perturbed Systems Using Accurate Reduced Model
}

\author{
Ayman E. M. Ahmed1, Mohamed Zohdy ${ }^{2}$ \\ ${ }^{1}$ Research Department, Egyptian Armed Force, Cairo, Egypt \\ ${ }^{2}$ Electrical and Systems Engineering, Oakland University, Rochester MI, USA \\ Email: ayman.ahmed@alumni.carleton.ca,zohdyma@oakland.edu
}

How to cite this paper: Ahmed, A.E.M. and Zohdy, M. (2021) Sliding Mode Control for Singularly Perturbed Systems Using Accurate Reduced Model. International Journal of Modern Nonlinear Theory and Application, 10, 1-12.

https://doi.org/10.4236/ijmnta.2021.101001

Received: November 22, 2020

Accepted: March 2, 2021

Published: March 5, 2021

Copyright $\odot 2021$ by author(s) and Scientific Research Publishing Inc. This work is licensed under the Creative Commons Attribution International License (CC BY 4.0).

http://creativecommons.org/licenses/by/4.0/

\begin{abstract}
In order to deal with unmodeled dynamics in large vehicle systems, which have an ill condition of the state matrix, the use of model order reduction methods is a good approach. This article presents a new construction of the sliding mode controller for singularly perturbed systems. The controller design is based on a linear diagonal transformation of the singularly perturbed model. Furthermore, the use of a single sliding mode controller designed for the slow component of the diagonalized system is investigated. Simulation results indicate the performance improvement of the proposed controllers.
\end{abstract}

\section{Keywords}

Singular Perturbation, Sliding Mode Control, Diagonalization Method

\section{Introduction}

The design requirements for high precision and high maneuverable missile, need it to be slender and long. Using light weight material and thin wall structure to design the motor for these long and thin missiles induced elasticity in the body. The rigid body model does not satisfy the needs of precision controller. Singular Perturbation analysis is used by many researchers to overcome such a problem [1] [2] [3] [4] where the fast state variables are the elastic forces and their time derivatives. Singular Perturbation theory first appeared in mathematical literature then in control literature. Many books and research papers are found in this direction, the most famous are the books by Kokotovic [3] and Nadiu [4].

The use of sliding mode control for singularly perturbed systems is explored. A number of articles have been published on this topic. Slotine [5] [6] proposed one of the earliest attempts by applying his sliding mode tracking control algorithm to a robot in singular perturbed form. Heck [7] also introduced sliding 
mode control for a singular perturbed system. Heck [7] used two sliding mode controllers, one for each of the fast and slow subsystems. Alvarez-Gallegos [1] introduced an approach to find a maximum value of the perturbation parameter $\varepsilon^{\star}$ when two sliding mode controllers are used for the full order singularly perturbed system. Alvarez-Gallegos [1] uses the Lyapunov approach that was introduced by Kokotovic [3]. These articles propose to design two separate sliding mode controllers for the fast and slow subsystems. The singularly perturbed system is separated into slow and fast modes by using quasi steady state techniques. The result is separate dynamic models for the fast and slow states, the literature proposed designing sliding mode controllers for the slow and fast modes. The difficulty with this method is that the quasi steady state model is only an approximation of the dynamics of the true full order systems.

We propose, in this article, to transform the full order singularly perturbed system into block diagonal form [8]. Based on the transformed systems we construct the slow and fast sliding mode controllers. We compare the performance of the sliding mode controller designed based on the quasi steady state approximation of the dynamics to the sliding mode controllers designed based on the block diagonal transformation. It is shown that sliding mode control design based on the transformed system has superior performance.

We also investigate the design of a single sliding mode controller for the full order system. This single sliding mode controller is designed based on the slow dynamics of the transformed system, the fast dynamics are considered as unmodeled high frequency dynamics.

The outline of this paper is as follows. Section 2 describes the singularly perturbed system and the appropriate transformations. Section 3 illustrates the sliding mode controller design for both reduced subsystems obtained using the diagonalization method. Section 4 describes the design of a single sliding mode controller for the full order system. Section 5 shows the simulation results.

\section{Problem Formulations}

The system considered in this paper may be represented in the following form,

$$
\begin{aligned}
& \dot{x}=A_{11} x+A_{12} z+B_{1} u \\
& \varepsilon \dot{z}=A_{12} x+A_{22} z+B_{2} u
\end{aligned}
$$

where $x \in R^{n}, \quad z \in R^{m}, u \in R^{r}$ and $\varepsilon$ is a small positive parameter. This is a form for standard linear time invariant singularly perturbed systems. Due to the high dimensionality of the full order system, singular perturbation theory is useful in decoupling the system into two reduced order subsystems considering the fact that fast modes are important only during a short initial period. After that period they are negligible and the behavior of the system can be described by its slow modes. The model with fast modes neglected is called the quasi steady state model (or zeroth order model) [3] [9]. Neglecting the fast modes is equivalent to assuming that they are infinitely fast, that is setting the perturbation parameter $\mathcal{E}$ in (2) to zero. Without fast modes the system described by equation (1) and (2) 
are reduced to,

$$
\begin{gathered}
\dot{\tilde{x}}_{1}=A_{11} \tilde{x}+A_{12} \tilde{z}+B_{1} \tilde{u} \\
0=A_{21} \tilde{X}_{1}+A_{22} \tilde{z}+B_{2} \tilde{u}
\end{gathered}
$$

where $\tilde{x}, \tilde{z}$ and $\tilde{u}$ are tracking vector error.

Thus we can get the following reduced system [9] [10],

$$
\dot{x}_{s}=A_{0} X_{s}+B_{0} u
$$

where $A_{0}=A_{11}-A_{12} A_{22}^{-1} A_{21}$ and $B_{0}=B_{1}-A_{12} A_{22}^{-1} B_{2}$

The system (5) is called the zeroth order model and is an approximation of the slow varying state of the full order system. Assuming that the slow variables are constant during fast transients i.e. $\tilde{z}=0$ and $\tilde{x}_{1}=x_{s}=$ constant thus the approximated fast subsystem for the system given by Equation (1) and (2) can be defined as,

$$
\varepsilon \dot{x}_{f}=A_{22} x_{f}+B_{2} u_{f}
$$

The eigenvalues of $A_{0}$ are good approximations of the slow modes of the full order system for sufficiently small $\varepsilon$ [1] [11]. It is possible to design a controller based on the quasi steady state model to stabilize the full order system for sufficiently small $\varepsilon$. However, for real systems the perturbation parameter $\varepsilon$ is not zero. Validation problems for the zero order model arise where for a certain value of $\varepsilon$, the zero order model can be stable while the slow modes of the original system are unstable [1] for this value of perturbation parameter $\varepsilon$. To overcome this problem, we introduce the diagonalization method [11]. This can be achieved by using two stage linear transformations. The first stage is to use the transformation,

$$
\dot{x}_{f}=z+L(\varepsilon) x
$$

This transforms the system described by Equations (1) and (2) into upper block diagonal form as shown in Equation (8)

$$
\left[\begin{array}{c}
\dot{x} \\
\dot{x}_{f}
\end{array}\right]=\left[\begin{array}{cc}
A_{11}-A_{12} L & A_{12} \\
R(L) & A_{22}+\varepsilon L A_{12}
\end{array}\right]\left[\begin{array}{c}
x \\
x_{f}
\end{array}\right]+\left[\begin{array}{c}
B_{1} \\
B_{2}+\varepsilon L B_{1}
\end{array}\right] u
$$

We required that matrix $L$ satisfy

$$
R(L)=A_{21}-A_{22} L+\varepsilon L A_{11}-\varepsilon L A_{12} L=0
$$

Then using the second stage of transformation

$$
x_{s}=x_{1}-M x_{f}
$$

To obtain,

$$
\left[\begin{array}{c}
\dot{x}_{s} \\
\dot{x}_{f}
\end{array}\right]=\left[\begin{array}{cc}
A_{s} & S(M) \\
0 & A_{f}
\end{array}\right]\left[\begin{array}{c}
x_{s} \\
x_{f}
\end{array}\right]+\left[\begin{array}{c}
B_{s} \\
B_{f}
\end{array}\right] u
$$

where $B_{s}=B_{1}-M B_{2}-\varepsilon M L B_{1}, \quad B_{f}=B_{2}+\varepsilon L B_{1}, \quad A_{s}=A_{11}-A_{12} L$ and $A_{f}=A_{22}+\varepsilon L A_{12}$, also we required that $n \times m$ matrix $M$ satisfies the linear algebraic equation, 


$$
S(M)=\varepsilon\left(A_{11}-A_{12} L\right) M-M\left(A_{22}+\varepsilon L A_{12}\right)+A_{12}=0
$$

Thus the system has the required block diagonal or decoupled form,

$$
\left[\begin{array}{l}
\dot{x}_{s} \\
\dot{x}_{f}
\end{array}\right]=\left[\begin{array}{cc}
A_{s} & 0 \\
0 & A_{f}
\end{array}\right]\left[\begin{array}{l}
x_{s} \\
x_{f}
\end{array}\right]+\left[\begin{array}{c}
B_{s} \\
B_{f}
\end{array}\right] u
$$

where the slow and the fast variables $x_{s}$ and $x_{f}$ can be solved independently of each other. The eigenvalues of $A_{s}$ are exactly the same as the slow poles of the original system. Similarly, the eigenvalues of $A_{f}$ are the same as the fast poles of the full order model. The matrix $L$ is dependent on the perturbation parameter, $\varepsilon$. Letting $\varepsilon \rightarrow 0$ gives us $L=L_{0}=A_{22}^{-1} A_{21}$ which transforms the diagonal model into the same as the quasi steady state model $\left(A_{s}=A_{0}\right)$. Changing the value of the perturbation parameter $\varepsilon$ to a considerable value and checking the difference between the eigenvalues of $A_{s}$ and $A_{0}$ gives us an indication if the quasi steady state is a valid approximation or not. One may expected that if $A_{s}$ is very sensitive to the value of $\varepsilon$ then using $A_{0}$ instead of $A_{s}$ may cause large errors in the approximation of the slow poles of the original system. Similarly, if $A_{21}$ and $A_{22}$ are dependent on $\mathcal{E}$, the approximation of the fast system where we put $A_{f}=A_{22}$ may lead to an unstable system or increase the steady state error of the closed loop system.

Based on the pervious analysis the diagonalization method must be used instead of quasi steady state to decouple the full order system especially when the quasi steady state fails to describe the stability properties of the full order model correctly.

Equation (9) and (13) is an asymmetric Riccati equation which need special procedure to be solved reader can found more detail in the literature [9] [10].

\section{Sliding Mode Controller Design}

In this section we will design sliding mode controllers based on the reduced subsystems obtained from transforming the systems into block diagonal form. This is different from the methods proposed in the literature [6] [12] [13] [14] [15] that design the sliding mode controllers based on the quasi steady state approximation of the slow and fast modes. The design of the sliding mode control for the full order system is done in two stages. First, design a slow controller based on the slow subsystem. Second, design fast controllers based on fast subsystem, then, use the composite of these controllers to control the full order model.

\subsection{Slow Sliding Mode Control Design}

The slow sliding mode controller is designed using the slow reduced order model in (14). The equivalent control method [3] is used to design such a controller. We define the linear switching surface as $S_{s}=C_{s} X_{s}$. The equivalent control law ensures that the system remains on the sliding surface and it has the form, 


$$
u_{e q}=-\left[\left(C_{s} B_{s}\right)^{-1} C_{s} A_{s}\right] x_{s}
$$

Using this control law in the slow subsystem obtained from the block diagonalized reduced system (14) we then obtained,

$$
\dot{x}_{\text {eqs }}=\left[I-B_{s}\left(C_{s} B_{s}\right)^{-1} C_{s}\right] A_{s} x_{S}
$$

To guarantee local stability for this controller we must ensure that the eigenvalues of the equivalent system given by equation (16) have negative real parts. A Lyapunov function is used to determine the discontinuous control law $\left(u_{N}\right)$ that will satisfy the reaching condition [7] [16]. This will ensure the global asymptotic stability of the closed loop system. The control law will have the form,

$$
u_{s}=u_{e q}+u_{N}=-\left[\left(C_{s} B_{s}\right)^{-1} C_{s}\right] A_{s} x_{s}-\left(C_{s} B_{s}\right)^{-1} \eta_{1} \operatorname{sgn}\left(S_{s}\right)
$$

where $u_{N}$ is a discontinuous control action that drives the state to the sliding surface.

\subsection{Fast Sliding Mode Controller Design}

The same argument used to design the slow sliding mode controller can be used to design the fast sliding mode controller. Define the linear fast switching surface $S_{f}=C_{f} X_{f}$. The equivalent control method is used to determine the control law for the fast subsystem given by Equation (14). The control law is given by,

$$
u_{f}=-\left[\left(C_{f} B_{f}\right)^{-1} C_{f}\right] A_{22} x_{f}-\left(C_{f} B_{f}\right)^{-1} \eta_{2} \operatorname{sgn}\left(S_{f}\right)
$$

which ensures global asymptotic stability for fast subsystem. The control law for the full order model will be the composite of the slow and fast controllers as follows,

$$
u=u_{s}\left(x_{s}\right)+u_{f}\left(x_{f}\right)
$$

Since the eigenvalues of the reduced order subsystems is the same as the full order model this means that the linear transformation preserves the stability condition for the closed loop system, and taking the stability analysis proposed by Kokotovic [3] into account, we could say that the full order model is globally stable for $\varepsilon \in\left[0, \varepsilon^{*}\right]$ almost everywhere given that $\varepsilon^{*}>0$.

\section{Slow Sliding Mode Controller for the Full Order Model}

In this section, a single sliding mode controller is proposed to control the full order model. The idea is to use the robust properties of the sliding mode controller to counter the effects of the unmodeled high frequency dynamics. We will consider that the unmodeled high frequency dynamics are represented by the fast subsystem. The main assumption is that the matrix $A_{22}$ is stable while the full order model given by Equation (1) and Equation (2) may be unstable at some specific value of $\mathcal{E}$. The surface parameter for the single sliding mode controller is chosen based on Slotine's [6] [17] [18] approach. The sliding surface 
has the form,

$$
S_{J}=\left(\frac{\mathrm{d}}{\mathrm{d} t}+\lambda\right)^{n-1} x_{s}
$$

where $\lambda$ is the bandwidth of the system [5] and is taken to be smaller than the lowest frequency of the fast subsystem. The control law is chosen based on an equivalent control method and has the following form,

$$
u_{J}=-\left[\left(\frac{\partial S_{J}}{\partial x_{s}} B_{s}\right)^{-1} \frac{\partial S_{J}}{\partial x_{s}}\right] A_{s} x_{s}-\left(\frac{\partial S_{J}}{\partial x_{s}} B_{s}\right)^{-1} \eta_{1} \operatorname{sgn}\left(S_{J}\right)
$$

An advantage of using the proposed controller is that the control law in this case will depends only on the slow state. As a result there is no need for measurement of the fast state, which is usually difficult to measure.

\section{Illustrative Example}

Sliding mode controller used usually in missiles and spacecraft [15] [19]. However, in analyzing the dynamics of flexible missiles, such as intermediate-range ballistic missiles or intercontinental ballistic missiles, it is convenient to use singular perturbation analysis. Since all detailed information on missiles is classified, we will use a simple example which suffers from flexibility to show the importance of our study.

Consider a magnetic tape control system [8] given by

$$
\begin{gathered}
{\left[\begin{array}{c}
\dot{x}_{1} \\
\dot{x}_{2} \\
\varepsilon \dot{z}_{1} \\
\varepsilon \dot{z}_{2}
\end{array}\right]=\left[\begin{array}{cccc}
0 & 0.4 & 0 & 0 \\
0 & 0 & 0.345 & 0 \\
0 & -0.524 & -0.465 & 0.262 \\
0 & 0 & 0 & -1
\end{array}\right]\left[\begin{array}{l}
x_{1} \\
x_{2} \\
z_{1} \\
z_{2}
\end{array}\right]+\left[\begin{array}{l}
0 \\
0 \\
0 \\
1
\end{array}\right] u} \\
y=\left[\begin{array}{cccc}
1 & 0 & 0 & 0 \\
0 & 0 & 1 & 0
\end{array}\right]\left[\begin{array}{c}
x_{1} \\
x_{2} \\
z_{1} \\
z_{2}
\end{array}\right]
\end{gathered}
$$

This can be put in standard singularly perturbed form as,

$$
\begin{gathered}
\dot{x}=A_{11} x+A_{12} z+B_{1} u \\
\varepsilon \dot{z}=A_{12} x+A_{22} z+B_{2} u
\end{gathered}
$$

where

$$
\begin{aligned}
& A_{11}=\left[\begin{array}{cc}
0 & 0.4 \\
0 & 0
\end{array}\right], A_{12}=\left[\begin{array}{cc}
0 & 0 \\
0.345 & 0
\end{array}\right], \\
& A_{12}=\left[\begin{array}{cc}
0 & -0.524 \\
0 & 0
\end{array}\right], A_{22}=\left[\begin{array}{cc}
-0.465 & 0.262 \\
0 & -1
\end{array}\right] \\
& B_{1}=\left[\begin{array}{l}
0 \\
0
\end{array}\right], B_{2}=\left[\begin{array}{l}
0 \\
1
\end{array}\right] \text { and } \varepsilon=0.1
\end{aligned}
$$

The first $2 \times 2$ transformation matrix $L$ has to satisfy,

$$
R(L)=A_{21}-A_{22} L+\varepsilon L A_{11}-\varepsilon L A_{12} L=0
$$


This gives us the value of $L$ as,

$$
L=\left[\begin{array}{cc}
0 & 1.2412 \\
0 & 0
\end{array}\right]
$$

The second $2 \times 2$ transformation matrix $M$ has to satisfy

$$
S(M)=\varepsilon\left(A_{11}-A_{12} L\right) M-M\left(A_{22}+\varepsilon L A_{12}\right)+A_{12}=0
$$

Similarly this gives us the value of $M$ as,

$$
M=\left[\begin{array}{cc}
0.0862 & 0.0325 \\
-0.9094 & -0.2489
\end{array}\right]
$$

The slow subsystem is given by,

$$
\dot{x}_{s}=A_{s} x_{s}+B_{s} u_{s}
$$

where $A_{s}=A_{11}-A_{12} L=\left[\begin{array}{cc}0 & 0.4 \\ 0 & -0.4282\end{array}\right]$ and $B_{s}=\left[\begin{array}{c}-0.0325 \\ 0.2489\end{array}\right]$

The fast subsystem can be described by,

$$
\dot{x}_{f}=A_{f} x_{f}+B_{f} u_{f}
$$

where $A_{f}=\left[\begin{array}{cc}-0.4222 & 0.262 \\ 0 & -1\end{array}\right]$ and $B_{f}=\left[\begin{array}{l}0 \\ 1\end{array}\right]$

We notice that the eigenvalues of the reduced system are the same as the eigenvalues of the full order model. We design two sliding mode controllers based on the slow and fast subsystems. For the slow subsystem the control law will be,

$$
u_{s}=\left[\begin{array}{ll}
0 & 0.4442
\end{array}\right] x_{s}-4.839 \operatorname{sgn}\left(S_{s}\right)
$$

For the fast subsystem the control law is,

$$
u_{s}=\left[\begin{array}{ll}
-1.2665 & -0.214
\end{array}\right] x_{f}-2 \operatorname{sgn}\left(S_{f}\right)
$$

The control law for the full order model will be the composite of the slow and fast controls as follows:

$$
u=u_{s}\left(x_{s}\right)+u_{f}\left(x_{f}\right)
$$

Figure 1 illustrates the closed loop time response of the full order system. It is observed that the absolute value of the error between the desired response and zeroth model response is larger than the absolute error between the desired response and the proposed model response based on diagonalization. The difference in the error can be four times larger.

Since the fast subsystem is stable then based on the decoupled reduced order system we will design a sliding mode controller for the slow subsystem. The fast subsystem will be considered as unmodeled high frequency dynamics. Choosing the surface parameter as,

$$
C_{s}=\left[\begin{array}{ll}
2 & 1
\end{array}\right]
$$

the control law will have the form,

$$
u_{s}=\left[\begin{array}{ll}
0 & 2.0217
\end{array}\right] x_{s}-5.4337 \operatorname{sgn}\left(S_{s}\right)
$$

Figure 2 illustrates the closed loop time response of the full order system using 


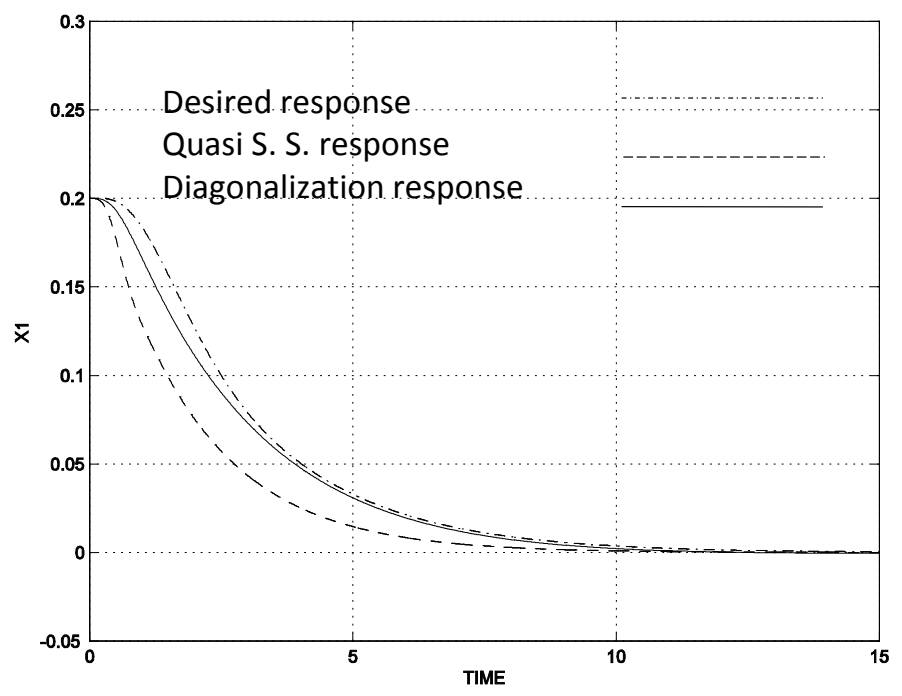

(a)

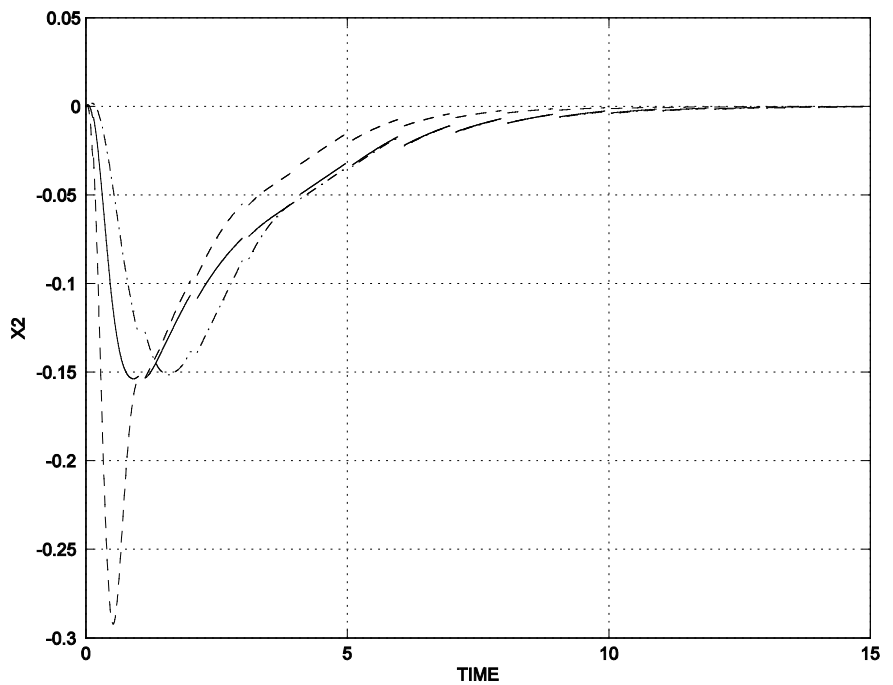

(b)

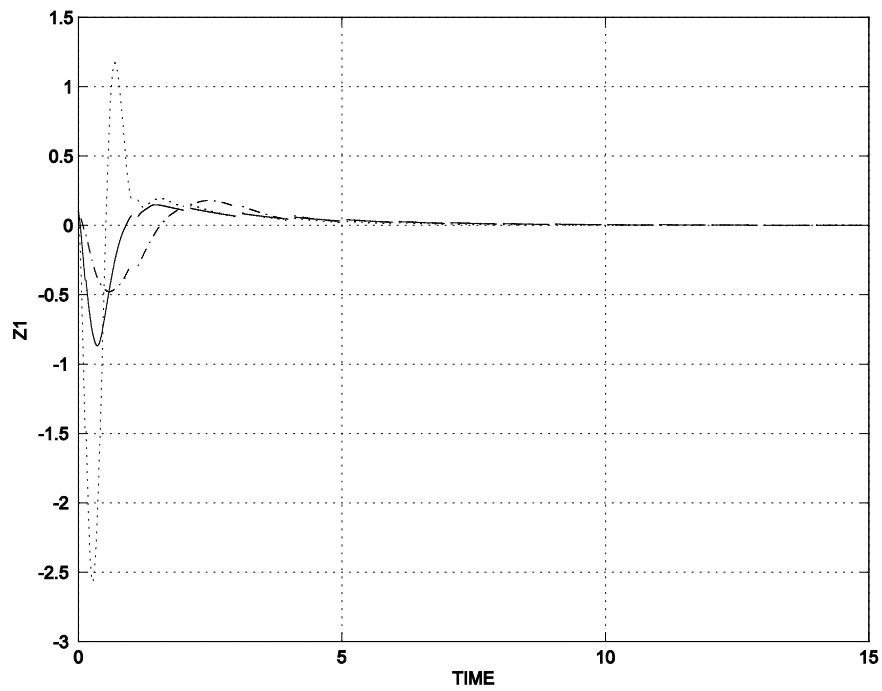

(c) 


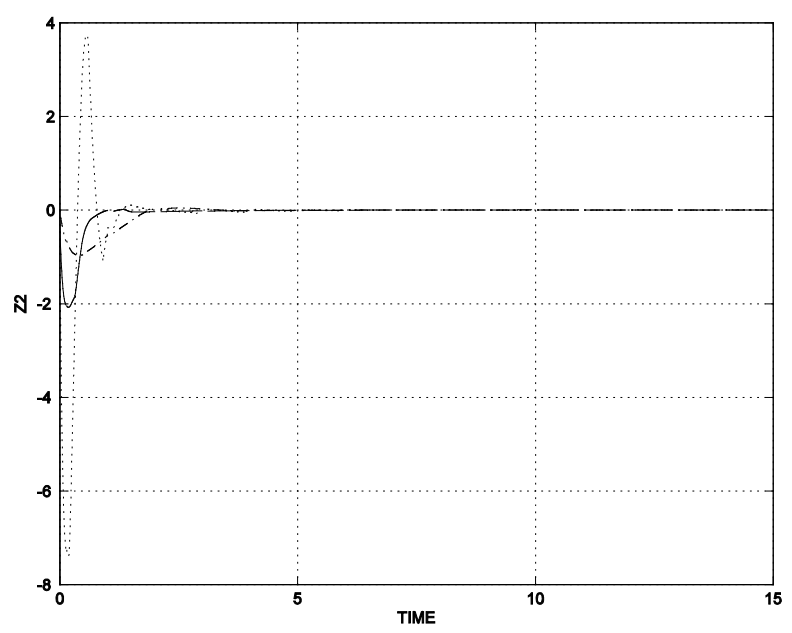

(d)

Figure 1. (a) Full order closed loop response for two sliding mode controller (Slow state); (b) Full order closed loop response for two sliding mode controller (Slow state); (c) Full order closed loop response for two sliding mode controller (Fast state); (d) Full order closed loop response for two sliding mode controller (Fast state).

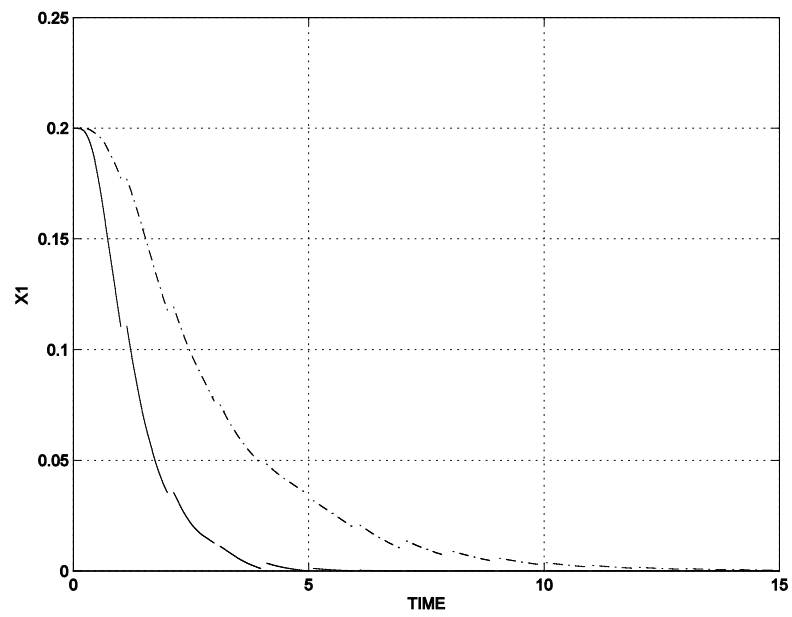

(a)

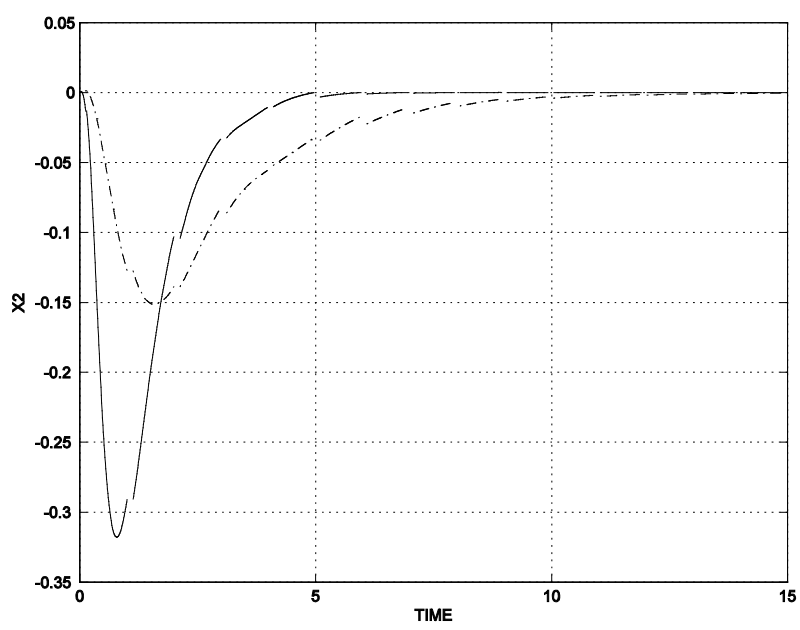

(b) 


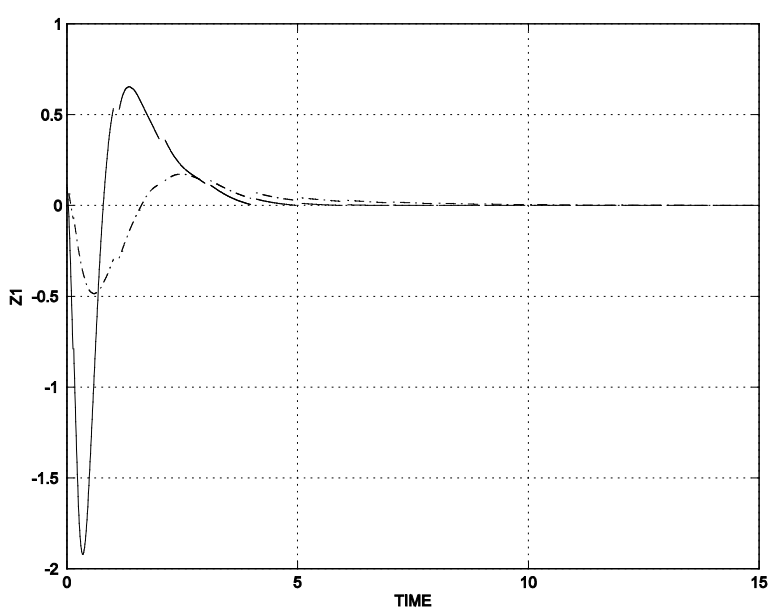

(c)

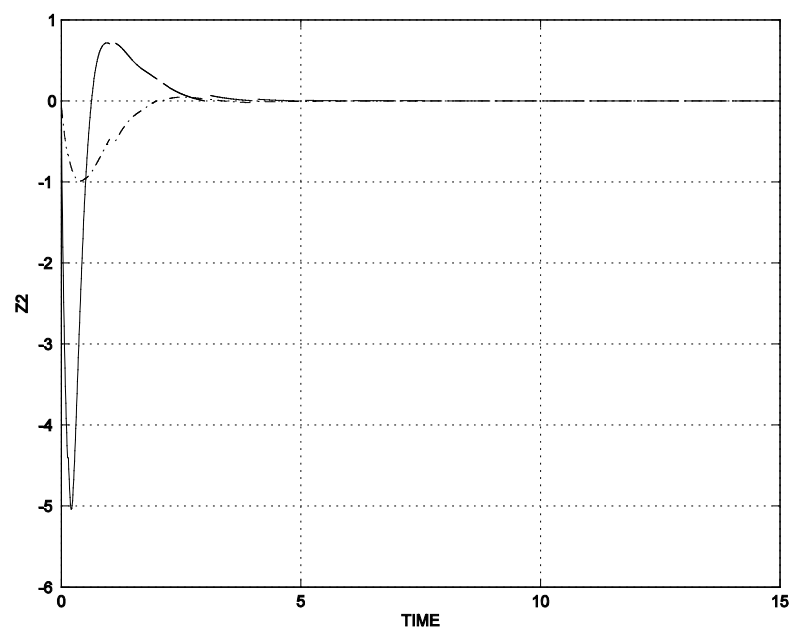

(d)

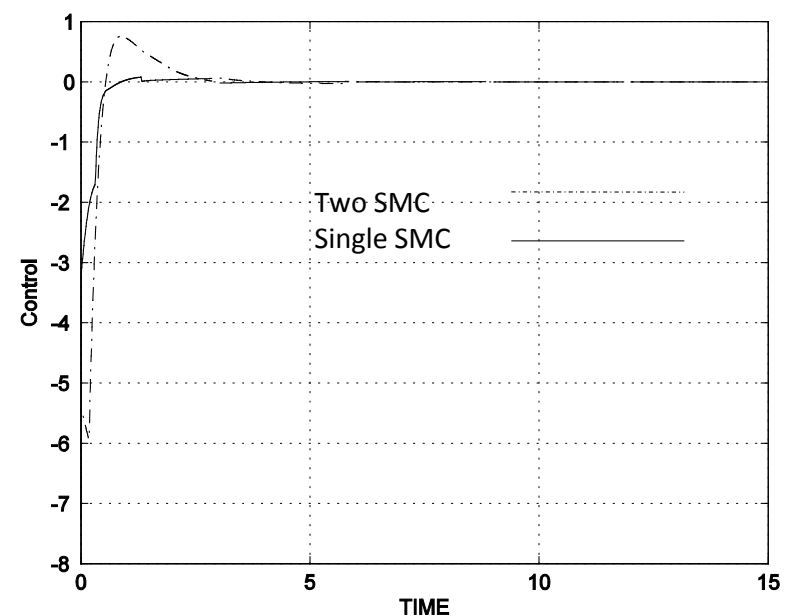

(e)

Figure 2. (a) Full order closed loop response for single sliding mode controller (Slow state); (b) Full order closed loop response for single sliding mode controller (Slow state); (c) Full order closed loop response for single sliding mode controller (Fast state); (d) Full order closed loop response for single sliding mode controller (Fast state); (e) Full order closed loop response for Two and single sliding mode controller (Control Signal). 
a single sliding mode controller. It is shown that it is possible to use one sliding mode controller to control the full order model for singularly perturbed system given that the fast subsystem is stable and we choose the right surface parameters that do not cause excitation of the fast subsystem.

\section{Conclusion}

Two sliding mode controller designs for singularly perturbed systems have been proposed. The designs are based on a block diagonal transformation of the system into fast and slow subsystems. The first design method proposes using two separate sliding mode controllers, one for the slow subsystem and a second for the fast subsystem. Simulation results indicate improved performance in comparison to previously published design methods since the errors which are produced during the decoupled process have been avoided. In the second proposed design method a single sliding mode controller is designed only for the slow subsystem and the fast subsystem is considered as high frequency unmodeled dynamics. These allow us to avoid measuring fast state, which is usually difficult to measure. Simulation results indicate good performance where the proposed controllers have less control effort compare to dual controller used before.

\section{Conflicts of Interest}

The authors declare no conflicts of interest regarding the publication of this paper.

\section{References}

[1] Alvarez-Gallegos, J. and Silva-Navarro, G. (1997) Two-Time Scale Sliding Mode Control for a Class of Nonlinear Systems. International Journal of Robust and Nonlinear Control, 7, 865-879. https://doi.org/10.1002/(SICI)1099-1239(199709)7:9\%3C865::AID-RNC240\%3E3.0. $\underline{\mathrm{CO} ; 2-0}$

[2] De Leon-Morales, J., Alvarez-Leal, J.G., Castro-Linares, R. (1997) Speed and Position Control of Flexible Joint Robot Manipulator via a Nonlinear Control-Observer Scheme. Proceeding of the IEEE International Conference on Control Applications, Hartford, 5-7 October 1997, 312-317. https://doi.org/10.1109/CCA.1997.627565

[3] Kokotovic, P., Khalil, H.K. and O’Reilly, J. (1999) Singular Perturbation Methods in Control, Analysis and Design. Society for Industrial and Applied Mathematics, Academic Press, Philadelphia. https://doi.org/10.1137/1.9781611971118

[4] Naidu, D.S. (1988) Singular Perturbation Methodology in Control Systems. IEE Series Vol. 34, Peter Peregrinus.

[5] Slotine, J.J. (1984) Sliding Controller Design for Non-Linear System. International Journal of Control, 40, 421-434. https://doi.org/10.1080/00207178408933284

[6] Slotine, J.J. and Hong, S. (1986) Two Time Sliding Control of Manipulators with Flexible Joints. Proceeding of 1986 American Control Conference, Seattle, 18-20 June 1986, 805-810. https://doi.org/10.23919/ACC.1986.4789045

[7] Heck, B.S. (1991) Sliding Mode Control for Singularly Perturbed Systems. International Journal of Control, 53, 985-1001. https://doi.org/10.1080/00207179108953660 
[8] Chow, J.H. and Kokotovic, P.V. (1976) A Decomposition of Near Optimum Regulators for System with Slow and Fast Modes. IEEE Transaction, 21, 701-705. https://doi.org/10.1109/TAC.1976.1101342

[9] Phillips, R.G. (1983) The Equivalence of Time Scale Decomposition Techniques Used in the Analysis and Design of Linear Systems. International Journal of Control, 37, 1239-1257. https://doi.org/10.1080/00207178308933043

[10] Narasimhamurthi, N. and Wu, F.F. (1977) On the Riccati Equation Arising from the Study of Singularly Perturbed Systems. Proceeding of Joint Automatic Control Conference, San Francisco, June 22-24 1977, 1244-1247.

[11] Khasawneh, B., Sabra, M. and Zohdy, M.A. (2014) Paralleled DC-DC Power Converters Sliding Mode Control with Dual Stages Design. Journal of Power and Energy Engineering, 2, 1-10. http://dx.doi.org/10.4236/jpee.2014.22001

[12] Hung, J.Y., Gao, W. and Hung, J.C. (1993) Variable Structure Control: A Survey. IEEE Transaction on Industrial Electronics, 40, 2-22. https://doi.org/10.1109/41.184817

[13] Li, T.S. (1995) Composite Sliding Mode Control of Singularly Perturbation Systems. Proceeding of the American Control Conference, Seattle, 21-23 June 1995, 2248 2249.

[14] Zohdy, M.A., Fadali, M.S. and Liu, J. (1995) Variable Structure Dynamic Output Feedback. Proceedings of 1995 American Control Conference, Seattle, 21-23 June 1995, 1518-1522. https://doi.org/10.1109/ACC.1995.521005

[15] Jia, S., Jia, Y., Xu, S. and Hu, Q. (2018) Maneuver and Active Vibration Suppression of Free-Flying Space Robot. IEEE Transactions on Aerospace and Electronic Systems, 54, 1115-1134

[16] Utkin, V.I. (1977) Variable Structure Systems with Sliding Mode. IEEE Transaction on Automatic Control, 22, 212-222. https://doi.org/10.1109/TAC.1977.1101446

[17] Nair, S.V., Lakhekar, G.V., Panchade, V.M. (2016) Fuzzy Sliding Mode Control Approach for Two Time Scale System: Stability Issues. 201610 th International Conference on Intelligent Systems and Control, Coimbatore, 7-8 January 2016, 1-8. https://doi.org/10.1109/ISCO.2016.7727081

[18] Wang, Y., Gao, Y., Karimi, H.R., Shen, H. and Fang, Z. (2018) Sliding Mode Control of Fuzzy Singularly Perturbed Systems with Application to Electric Circuit. IEEE Transactions on Systems, Man, and Cybernetics, 48, 1667-1675. https://doi.org/10.1109/TSMC.2017.2720968

[19] Zhang, Y., Zhao, Y. (2014) A Higher-Order Sliding Mode Controller Design for Spacecraft Attitude Tracking System. 2014 International Conference on Mechatronics and Control, Jinzhou, 3-5 July 2014, 1248-1251. https://doi.org/10.1109/ICMC.2014.7231752 